\title{
Five-year survivor with liver metastasis from gastric cancer successfully treated with systemic chemotherapy
}

\author{
Hiroki Saitoh, Narikazu Boku, Atsushi Ohtsu, Shuichi Hironaka, Shinichi Miyamoto, Yasuo Hamamoto, \\ Masana OHKuWA, and Shigeaki Yoshida \\ Division of Digestive Endoscopy and Gastrointestinal Oncology, National Cancer Center Hospital East, 6-5-1 Kashiwanoha, Chiba 277-8557, \\ Japan
}

\begin{abstract}
We report a patient with liver metastasis from gastric cancer who has achieved a 5-year survival after systemic chemotherapy. The patient was diagnosed with advanced gastric cancer and received a total gastrectomy in August 1991, followed by adjuvant chemotherapy. Liver and lymph node metastases were detected in April 1994, and systemic chemotherapy with a combination of etoposide, doxorubicin, and cisplatin was initiated. Although the liver metastasis completely disappeared, lymph node metastasis at the falciform ligament of the liver and around the portal fissure remained after six courses of this therapy. A second type of chemotherapy, a combination of 5-fluorouracil and methotrexate, was then administered, 12 times, from December 1994 to May 1995, during which time no disease progression was observed. After surgery for the metastatic lymph nodes in August 1995, no progression was observed until December 1998, when a tumor thrombus was detected in the portal vein. Combination chemotherapy of irinotecan and cisplatin was initiated in January 1999. Although tumor regression was achieved after two courses of this, the disease continued to progress after five courses. In July 1999, a fourth type of chemotherapy, using $1 \mathrm{M}$ tegafur- $0.4 \mathrm{M}$ gimestat-1 $\mathrm{M}$ otastat potassium (S-1), was initiated, and size reduction of the tumor thrombus was achieved; this therapy has continued to the time of submission of this report.
\end{abstract}

Key words Five-year survivor - Gastric cancer · Alpha fetoprotein $\cdot$ Liver metastasis

\section{Introduction}

Although the prognosis of patients with unresectable or recurrent gastric cancer is poor, with the median survival time being about 7 months, a survival benefit of systemic chemotherapy for unresectable or advanced

Offprint requests to: $\mathrm{N}$. Boku

Received: April 3, 2000 / Accepted: June 27, 2000 gastric cancer has been shown by several phase II studies [1-3]. In 226 patients enrolled in phase II studies by the Gastrointestinal Oncology Study Group of the Japan Clinical Oncology Group, the 5-year survival rate was $4 \%$ (8 patients) [4]. Almost all of these 5-year survivors had lymph node metastasis as an unresectable factor, and only 1 patient had liver metastasis. Seven of these eight 5-year survivors achieved complete or partial remission after first-line chemotherapy. Thus, it appears that response to first-line chemotherapy may contribute to cure and long-term survival.

Here, we report a patient with liver metastasis from gastric cancer who has survived for more than 5 years because of successful systemic chemotherapy.

\section{Case report}

The patient was 57 years old when he received a total gastrectomy for advanced gastric cancer in August 1991. The tumor was type 2 according to the macroscopic classification of the Japanese classification of gastric carcinoma [5], and was located in the middle part of the gastric body. Histological examination of the resected specimen revealed that the tumor was a poorly differentiated adenocarcinoma invading the muscularis propria, with perigastric lymph node metastasis. The patient received adjuvant chemotherapy with oral tegaful and uracil (UFT; $600 \mathrm{mg} /$ day) for 8 months after surgery.

Abdominal lymph node metastasis and a liver metastasis ( $4 \times 4 \mathrm{~cm}$ in size), were detected in April 1994 (Fig. 1a), with the patient showing a raised serum level of alpha-fetoprotein (AFP), of $300 \mathrm{ng} / \mathrm{ml}$ (normal level, less than $10 \mathrm{ng} / \mathrm{ml}$ ). Combination chemotherapy, consisting of etoposide $\left(100 \mathrm{mg} / \mathrm{m}^{2}\right.$, days 4,5 , and 6$)$, doxorubicin $\left(20 \mathrm{mg} / \mathrm{m}^{2}\right.$, days 1 and 7$)$, and cisplatin $\left(40 \mathrm{mg} / \mathrm{m}^{2}\right.$, days 2 and 8 ) (EAP) was then initiated, and repeated every 4 weeks (Fig. 2). The liver metastasis 
a,b
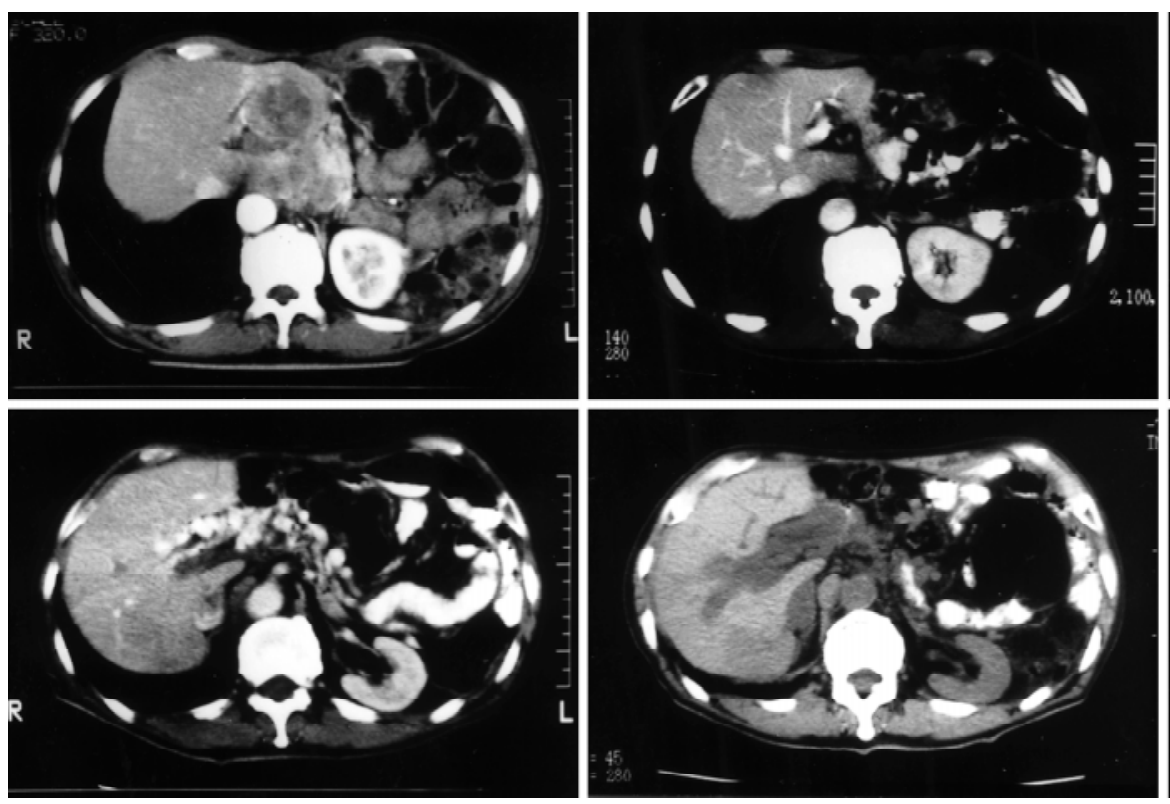

d,e

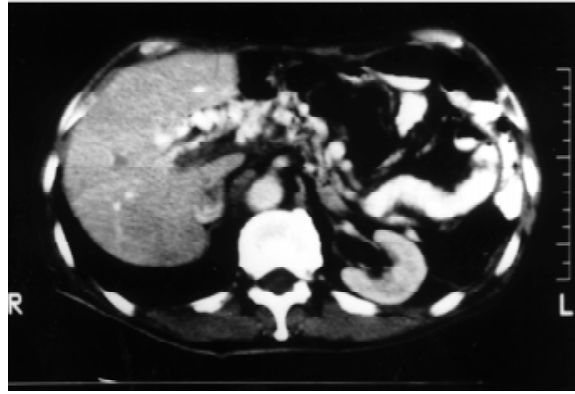

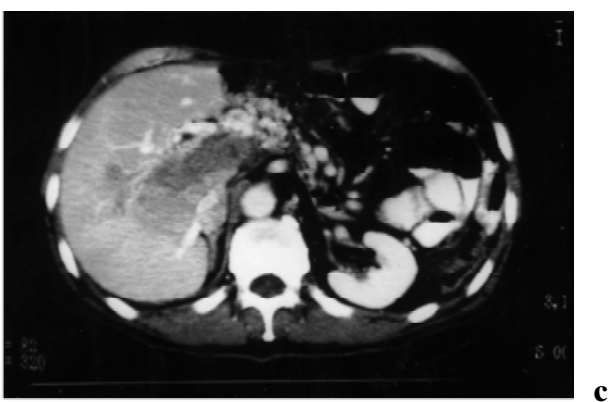

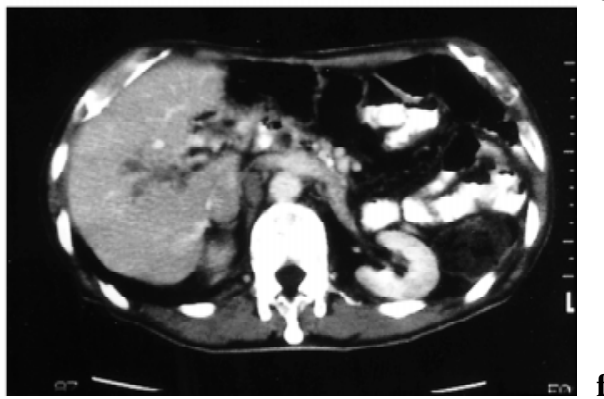

tumor thrombus was reduced after combined chemotherapy

Fig. 1a-f. Abdominal computed tomographic scans of the patient. a In April 1994, recurrence was identified in the left lobe of the liver and in lymph node after surgical resection. $\mathbf{b}$ In December 1994, after six courses of etoposide, doxorubicin, cisplatin (EAP) therapy, the liver metastasis had completely disappeared. c In December 1998, a tumor thrombus was identified in the portal vein. d In may 1999, the size of the with irinotecan and cisplatin. e In July 1999, tumor progression was observed after five courses of irinotecan and cisplatin (before the initiation of $1 \mathrm{M}$ tegafur- $0.4 \mathrm{M}$ gimestat- $1 \mathrm{M}$ otastat potassium [S-1] therapy). f In August 1999, the tumor thrombus was reduced in size after the initiation of S-1 therapy

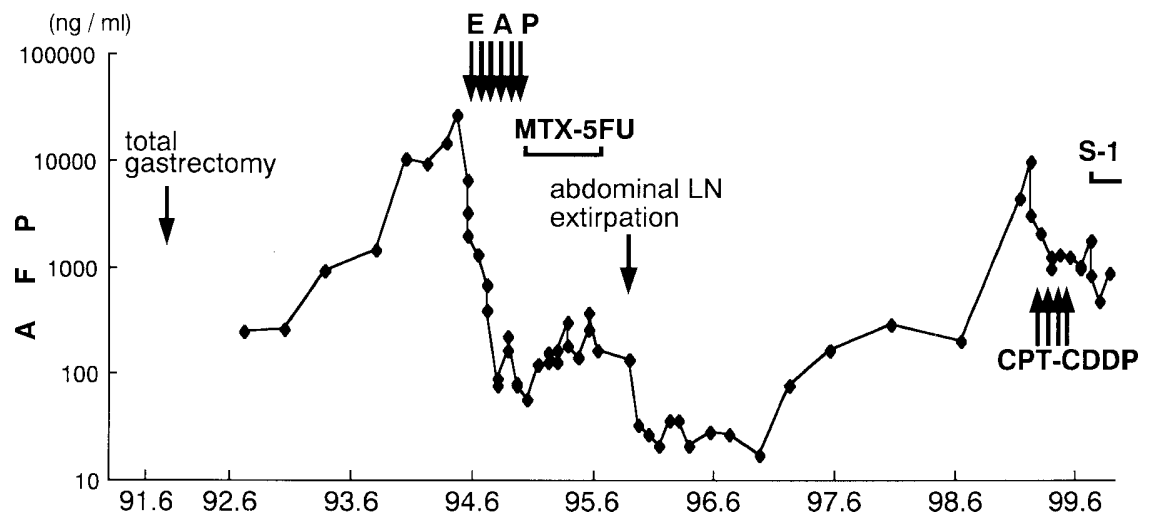

Fig. 2. Time course of the patient's alpha-fetoprotein $(A F P)$ levels. $M T X$ $5 F U$, methotrexate and 5-fluorouracil with leucovorin; $L N$, lymph node; $C P T-C D D P$, irinotecan and cisplatin

completely disappeared (Fig. 1b), and partial remission of the lymph node metastasis was achieved. In November 1994, after the completion of six courses of EAP chemotherapy, AFP serum levels remained above normal limits $(57.4 \mathrm{ng} / \mathrm{ml})$, and lymph node metastasis remained around the portal fissure.

A second type of chemotherapy, consisting of methotrexate $\left(100 \mathrm{mg} / \mathrm{m}^{2}\right.$ per day) and 5-fluorouracil $\left(600 \mathrm{mg} / \mathrm{m}^{2}\right)$ with leucovorin $\left(10 \mathrm{mg} / \mathrm{m}^{2}\right.$, six times $)$ (MTX/5-FU) administered weekly, was started in December 1994. However, this regimen was discon- tinued after 12 cycles because of patient refusal, due to nausea and vomiting. During this therapy, lymph node size and AFP serum levels showed no change.

Computed tomography (CT) detected increased lymph node size in July 1995. At surgery performed on August 10, 1995, several lymph nodes were macroscopically detected around the portal fissure; however, only four of them could be removed, because of severe fibrotic change at the hepatoduodenal ligament. Histologically, one of the four removed lymph nodes involved cancer cells. Although the patient's AFP 
serum levels gradually increased from March 1997, CT detected no lesions until December 1998, when a tumor thrombus was observed in the portal vein (Fig. 1c).

Combination chemotherapy of irinotecan $\left(70 \mathrm{mg} / \mathrm{m}^{2}\right.$, days 1 and 15$)$ and cisplatin $\left(80 \mathrm{mg} / \mathrm{m}^{2}\right.$, day 1$)$ was initiated from January 1999, and repeated every 4 weeks. Although the size of the tumor thrombus was reduced after the first course (Fig. 1d), tumor progression was observed after five courses had been completed, in July 1999 (Fig. 1e).

From July 1999, S-1 (1 M tegafur-0.4 M gimestat$1 \mathrm{M}$ otastat potassium; $80 \mathrm{mg} /$ body per day) was then administered orally, daily. However, this therapy was discontinued after only 2 weeks when grade 2 leukopenia, grade 4 thrombocytopenia, grade 2 stomatitis, and grade 2 diarrhea appeared. CT revealed a marked reduction in the size of the portal vein thrombus in August 1999 (Fig. 1f). S-1 therapy was then recommenced, with dose modification by administration every other week, and the partial remission has continued until the time of submission of this report, without reappearance of severe toxicity.

Therefore, this patient has survived for more than 5 years after the initiation of systemic chemotherapy against recurrence of gastric cancer.

All treatments were performed after the patient's informed consent was obtained.

\section{Discussion}

Some authors have proposed criteria for an AFPproducing gastric cancer, and it has been reported that patients with AFP-producing gastric cancer show poor prognosis after surgical resection. Although immunohistochemical staining of AFP was not performed in the present patient, the patient was diagnosed with an AFPproducing gastric cancer because of the markedly high serum levels of AFP at the time of recurrence, in the absence of other disease such as liver cirrhosis or hepatocellular carcinoma.

The use of the EAP combination chemotherapy for advanced gastric cancer was first reported by Preusser et al. [6] and showed a high response rate. Kelsen et al. [7] later reported that - compared with a combination of 5-fluorouracil, doxorubicin, and methotrexate EAP showed severe toxicity, and they noted that it was no longer commonly used. However, Gonda et al. [8] have since reported that EAP was highly effective against AFP-producing gastric cancer. When EAP was selected as chemotherapy after disease recurrence in the present patient, complete remission of liver metastasis and partial remission of abdominal lymph node metastasis was achieved. As it is well known that cisplatin can lead to neurotoxicity at doses higher than
$500 \mathrm{mg} / \mathrm{m}^{2}$, EAP was discontinued after the completion of six courses [9].

5-FU is a key antineoplastic agent against gastric cancer; sequential MTX/5-FU chemotherapy was first reported by Bertino et al. [10], and showed antitumor effects against gastric cancer [11,12]. A phase II study of sequential MTX/5-FU as a second-line chemotherapy showed long survival times, despite low response rates, and could be performed at outpatient clinics [13]. For these reasons, we selected sequential MTX/5FU chemotherapy as a second-line chemotherapy after the completion of EAP. The present patient showed not tumor progression during the period of this chemotherapy.

Seike et al. [14] reported the outcomes of 18 patients with advanced gastric cancer who underwent surgery after successful systemic chemotherapy. Among the 5-year survivors, it was found that EAP was the most common chemotherapy regimen and that lymph node metastasis was the only unresectable factor at the initiation of chemotherapy. The present patient underwent surgery for a second time for abdominal lymph node metastasis. Although only some of several lymph nodes could be removed macroscopically, no recurrence of lymph node metastasis has been detected since. There is considered to be no residual disease and no recurrence around the portal fissure.

In a phase II study of a combination chemotherapy of irinotecan and cisplatin, a high response rate was observed even in patients refractory to previous chemotherapies [15]. In the present patient, it had been more than 4 years since the completion of EAP, and he showed no neurotoxicity when the portal vein thrombus appeared. Thus, it was considered that a combination chemotherapy of irinotecan and cisplatin would be effective and tolerable. Reduction in size of the tumor thrombus in the portal vein was achieved after this therapy, without evidence of severe neurotoxicity.

$\mathrm{S}-1$ is a new oral fluoropyrimidine with high activity against gastric cancer, and with mild toxicity [16,17]; however, its activity in previously treated patients has not been clarified. Because S-1 has different antitumor mechanisms from that of sequential MTX/5-FU, it was selected as the fourth-line chemotherapy for the present patient. Although this therapy was interrupted 2 weeks after initiation because of severe toxicity, a reduction in size of the tumor thrombus in the portal vein was achieved. This therapy was recommenced with schedule modification without severe toxicity, and the patient's partial remission has continued to the time of submission of this article.

It is generally considered that systemic chemotherapy for unresectable or recurrent gastric cancer can prolong survival on an intention-to-treat basis; however, reports of 5-year survivals are still rare. Although there are 
some case reports of 5-year survivors [18,19], none of the randomized trials using systemic chemotherapy have recorded cases of 5-year survivors. Indeed, it has been estimated that the 5-year survival rate any be as low as $0.1 \%$ [20-23].

The chemotherapy regimens used in the present patient were chosen on the basis of the evidence mentioned above, and successfully resulted in a 5-year survival in spite of the presence of disease. Thus, successful systemic chemotherapy can contribute to long survival of gastric cancer patients with liver and lymph node metastasis.

\section{References}

1. Phyrhonen S, Kuitunen T, Nyandoto P, Kouri M. Randomized comparison of fluorouracil, epidoxorubicin and methotrexate (FAMTX) plus supportive or care with supportive care alone in patients with non-resectable gastric cancer. $\mathrm{Br} \mathrm{J}$ Cancer 1995;71:587-91.

2. Murad AM, Santiago FF, Petroianu A, Rocha PR, Rodrigues MA, Rausch M. Modified therapy with 5-fluorouracil, doxorubicin, and methotrexate in advanced gastric cancer. Cancer 1993;72:37-41.

3. Glrimelius B, Hoffman K, Haglund U, Nyren O, Sjoden PO. Initial or delayed chemotherapy with best supportive care in advanced gastric cancer. Ann Oncol 1994;5:189-90.

4. Ohkuwa M, Miyata Y, Ohtsu A, Boku N, Yoshida S, Shirao K, et al. Long-term results of patients with unresectable gastric carcinoma treated with chemotherapy in the Japan Clinical Oncology Group. Third international conference on biology, prevention and treatment of gastrointestinal malignancies. September 23-26, 1998 Cologne, Germany.

5. Japanese Research Society for Gastric Cancer. Japanese classification of gastric carcinoma. First English ed. Tokyo: Kanehara; 1993.

6. Preusser P, Wilke H, Achterrath W, Fink U, Lenaz L, Heiniche A, et al. Phase II study with the combination etoposide, doxorubicin and cisplatin in advanced measurable gastric cancer. J Clin Oncol 1989;7:1310-7.

7. Kelsen D, Atiq OT, Saltz L, Niedzwiechi D, Ginn D, Chapman D, et al. FAMTX versus etoposide, doxorubicin, and cisplatin: a random assignment trial in gastric cancer. J Clin Oncol 1992;10: $541-8$.

8. Gonda T, Ishida H, Higuchi T, Hirukawa H, Nakajima H, Hojo I, et al. A case of AFP (alpha-fetoprotein) producing gastric cancer successfully treated with EAP (etoposide, adriamycin, cisplatin) therapy (in Japanese). Gan To Kagaku Ryoho (Jpn J Cancer Chemother) 1994;21:1659-63.

9. van der Hoop RG, van der Burg ME, ten Bokkel Huinink WW, van Houwelingen C, Neijt JP. Incidence of neuropathy in 395 patients with ovarian cancer treated with or without cisplatin. Cancer 1990;66:1697-702.

10. Bertino JR, Sawicki WL, Lindquist CA, Gupta VS. Scheduledependent antitumor effects of methotrexate and 5-fluorouracil. Cancer Res 1977;37:327-8.
11. Bruckner HW, Cohen J. MTX/5-FU trials in gastro-intestinal and other cancers. Semin Oncol 1983;10:32-914.

12. Perez JE, Lacava JA, Diminguez ME, Rodriguez R, Barbieri MR, Ortiz EH, et al. Biochemical modulation of 5-fluorouracil by methotrexate in patients with advanced gastric carcinoma. Am J Clin Oncol 1998;21:452-7.

13. Yamamichi N, Shirao K, Hyodo I, Koizumi W, Seki S, Kawai K, et al. A phase II study of sequential chemotherapy of methotrexate and 5-fluorouracil for advanced gastric cancer as second line chemotherapy - JCOG trial (in Japanese). In: Yamaoka Y, editor. Proc. 33rd annual meeting of Japan Society for Cancer Treatment. Int J Clin Oncol 1995; Abstract 569.

14. Seike K, Ohtsu A, Yoshida S, Kinoshita T, Ono M, Koizumi W, et al. Efficacy of surgery after successful chemotherapy for advanced gastric cancer. Int J Clin Oncol 1998;3:159-64.

15. Boku N, Ohtsu A, Shimada Y, Shirao K, Seki S, Saito H, et al. Phase II study of a combination of irinotecan and cisplatin against metastatic gastric cancer. J Clin Oncol 1999;17:31923.

16. Sakata Y, Ohtsu A, Horikoshi N, Sugimachi K, Mitachi Y, Taguchi T. Late phase II study of novel oral fluoropyrimidine anticancer drug S-1 (1 M tegafur- $0.4 \mathrm{M}$ gimestat-1 M otastat potassium) in advanced gastric cancer patients. Eur J Cancer 1998;34:1715-20.

17. Kurihara M, Koizumi W, Hasegawa K, Nakano K. Late phase II study of S-1, a novel oral fluoropyrimidine derivative, in patients with advanced gastric cancer. Proc Am Soc Clin Oncol 1998; 17: Abstract 262.

18. Wada $\mathrm{T}$, Iwanaga $\mathrm{T}$, Koyama $\mathrm{H}$, Furukawa $\mathrm{H}$, Akita $\mathrm{N}$, Wada A, et al. A case report of long-term survival in advanced gastric cancer with multiple liver metastases treated with mitomycin $\mathrm{C}$ and tegafur therapy after palliative gastrectomy (in Japanese). Gan To Kagaku Ryoho (Jpn J Cancer Chemother). 1988;15:353-6.

19. Iwase K, Takenaka H, Sumimura J, Ishizaka T, Takagaki M, Bessho $\mathrm{T}$, et al. A 5-year-survival case in which complete response was recognized after combined chemotherapy using 5-fluorouracil, adriamycin and mitomycin C (FAM) for unresectable gastric cancer (in Japanese) Gan To Kagaku Ryoho (Jpn J Cancer Chemother) 1992;19:2235-7.

20. Wils JA, Klein HO, Wagener DJ, Bleiberg H, Reis H, Korsten F, et al. Sequential high-dose methotrexate and fluorouracil combined with doxorubicin - a step ahead in the treatment of advanced gastric cancer: a trial of the European Organization for Research and Treatment of Cancer Gastrointestinal Tract Cooperative Group. J Clin Oncol 1991;9:827-31.

21. Webb A, Cunningham D, Scarffe JH, Harper P, Norman A, Joffe JK, et al. Randomized trail comparing epirubicin, cisplatin, and fluorouracil versus fluorouracil, doxorubicin, and methotrexate in advanced esophagogastric cancer. J Clin Oncol 1997;15:2617.

22. Cullinan SA, Moertel CG, Wieand HS, O'Connell MJ, Poon MA, Krook JE, et al. Controlled evaluation of three drug combination regimens versus fluorouracil alone for the therapy of advanced gastric cancer. North Central Cancer Treatment Group. J Clin Oncol 1994;12:412-6.

23. Waters JS, Norman A, Cunnigham D, Scarffe JH, Webb A, Harper P, et al. Long-term survival after epirubicin, cisplatin and fluorouracil for gastric cancer: results of a randomized trial. Br J Cancer 1999;80:269-72. 